\title{
Epistemologia social e cultura digital: reflexões em torno das formas de escritas na web
}

\author{
Regina Maria Marteleto \\ Doutora; Universidade Federal do Rio de Janeiro (UFRJ), Rio de Janeiro, RJ, Brasil; \\ regina.marteleto@gmail.com
}

\begin{abstract}
Resumo: O artigo apresenta alguns eixos conceituais a fim de refletir sobre a produção, mediação e apropriação de conhecimentos, e sobre a configuração de dispositivos de informação e comunicação, no contexto de uma 'cultura digital'. Recupera a ideia de epistemologia social formulada por M. Egan e J. Shera, e atualizada por autores das áreas da informação e da filosofia, para refletir sobre como as formas epistêmicas relacionam-se às práticas sociais e institucionais. Exploram-se ainda alguns conceitos da linha francesa das Ciências da Informação e Comunicação: dispositivo infocomunicacional; cultura digital; texto; hipertexto; arquitexto, a fim de refletir sobre as formas estruturais das escritas e leituras na web. Se a epistemologia social orienta um programa de estudos sobre as questões de acesso, produção e apropriação social de conhecimentos, as pesquisas sobre as formas de escrita indicam caminhos teórico-práticos para entender e praticar as escritas na web.
\end{abstract}

Palavras-chave: Epistemologia social; Dispositivos de informação e comunicação; Cultura digital; Texto; Arquitexto

\section{Introdução}

As reflexões apresentadas nesse artigo sustentam-se em eixos teóricos e metodológicos empregados em pesquisas do Grupo 'Cultura e Processos Infocomunicacionais (Culticom)' cujo o objetivo geral é estudar os mecanismos de produção, mediação e apropriação de informações em redes sociais híbridas, isto é, aquelas que se originam nas relações entre pesquisadores, profissionais, grupos e movimentos sociais que compartilham objetivos comuns de mudança e participação social. Mais especificamente, trata-se de redes de mobilização e participação social que vêm se reconfigurando no contexto de uma cultura digital. Para tanto, três questões principais orientam os estudos a respeito dos modos de democratização do acesso e da expressão nos novos espaços digitais: a) as formas de leitu- 
ra, escrita e oralidade na Web garantem, para diferentes usuários, a multiplicidade de vozes e o amplo acesso a conteúdos qualificados e diversificados? b) qual o papel das redes, para os movimentos sociais e suas lutas por direitos políticos e sociais, como o da livre expressão e o da participação social? c) o emprego e a apropriação das informações e conhecimentos nas novas mídias digitais podem, além de fundamentar um pensamento crítico relativo às formas de produção de conhecimento, abrir espaços para interações entre atores diversos e suas diferentes formas de conhecer? Trata-se, então, de uma tentativa de compreender os espaços virtuais na perspectiva da relação entre seus atores, que são, por fim, qualificadores de saberes, informações e experiências.

Uma das fontes inspiradoras para as pesquisas sobre o tema consiste na noção de 'epistemologia social' elaborada por M. Egan e J. Shera, em meados do século 20. A partir da retomada das reflexões e visões ampliadas desses dois fundadores sobre o conhecimento e a informação enquanto produtos culturais que carregam as marcas da epistemologia social do seu tempo, o artigo recupera algumas atualizações da epistemologia social no campo da filosofia, a fim de complementar e ampliar as reflexões acerca das condições sociais e institucionais de produção de conhecimentos.

Em seguida e como fruto dos diálogos construídos no âmbito das atividades da Rede Franco-Brasileira de Pesquisadores em Mediações e Usos Sociais de Saberes e Informação (Rede Mussi), o artigo trata de abordagens recentes presentes na literatura francesa das Ciências da Informação e Comunicação (SIC) - dispositivos de informação e comunicação, cultura digital, texto e arquitexto - no intuito de eleger alguns conceitos úteis à compreensão dos processos de produção, mediação e apropriação de conhecimentos nos ambientes digitais.

Como consideração final desses apontamentos, afirma-se a validade da retomada dos termos teóricos, éticos e metodológicos da epistemologia social no estudo de novas formas de produzir, mediar e difundir conhecimentos para a sua apropriação social e informacional, valendo-se dos dispositivos digitais. Nesse sentido, as análises sobre os modos de existir dos 'seres culturais' no contexto da cultura digital poderiam fornecer elementos para a compreensão das (novas) formas de leituras, escritas e textualidades na web, construindo um caminho 
importante para a democratização, a apropriação e a criação de dispositivos de informação e comunicação no meio digital.

\section{Por que e como uma epistemologia social?}

O intento aqui é recuperar o sentido teórico e prático da epistemologia social, tal qual foi cunhada no campo de estudos e práticas da informação por Margareth Egan e Jesse Shera, com algumas atualizações de autores da filosofia. Em 1952 os dois autores publicaram em colaboração o artigo "Fundamentos de uma teoria da bibliografia", no qual, pela primeira vez, usou-se o termo "epistemologia social" que, segundo os autores, procura estudar como uma pessoa adquire conhecimento de outra em contextos sociais. Seu objetivo último seria [...] estudar quais são as práticas sociais que facilitam a aquisição do conhecimento." (SHERA, 1973, p. 89). Em outro trabalho em conjunto, reafirmavam a necessidade de uma aliança entre a organização, a classificação e a sociologia do conhecimento:

\footnotetext{
Mesmo um breve exame da história da classificação das ciências demonstra o quanto o esforço de organizar os conhecimentos depende da epistemologia social do tempo em que eles são produzidos. Essa dependência da teoria da classificação em relação ao estado da sociologia do conhecimento será confirmada ainda de maneira mais forte no futuro. (EGAN; SHERA, 1952 p. 82).
}

A epistemologia social teria um forte componente interdisciplinar, com foco na produção, fluxo, integração e consumo de todas as formas de pensamento comunicadas através da estrutura social. Para tanto, se valeria das tecnologias e do conhecimento, não apenas do sistema cognitivo dos indivíduos, mas também da rede comunicacional da sociedade, tendo ênfase nos seguintes aspectos:

a) problema da cognição - como os sujeitos conhecem;

b) problema da cognição social - como a sociedade conhece, e a natureza do sistema psicossocial por meio do qual o conhecimento pessoal transforma-se em conhecimento social, isto é, em conhecimento apropriado pela sociedade; 
c) a história e a filosofia do conhecimento, e como ele se desenvolveu ao longo do tempo numa variedade de culturas;

d) os mecanismos e sistemas bibliográficos existentes, e em qual medida eles se adéquam às realidades do processo comunicacional, às descobertas das pesquisas epistemológicas e ao conteúdo substantivo do próprio estoque de conhecimentos da sociedade (SHERA, 1973, p. 89).

Para Shera (1971) o estudo dos conhecimentos e da sua materialização informacional deveria estar baseado na seguinte triangulação: a) do atomismo, significando a operação tecnológica; b) do conteúdo, isto é, aquilo que é transmitido; c) do contexto social e cultural, o qual define as características dos dois primeiros aspectos. Em resumo, a reflexão e a prática da epistemologia social seriam uma espécie de programa ou de dever de ofício epistemológico, ético e político para os produtores, os mediadores e os consumidores dos produtos da cultura, do conhecimento, da informação e da comunicação.

Nas últimas décadas diversos estudiosos da epistemologia social têm se dedicado a dimensionar a sua abrangência e o elenco de suas questões. No campo de estudos da informação, Zandonade (2004) reafirma a atualidade de uma epistemologia social no ordenamento informacional da realidade social, política e econômica em nível mundial, ao afirmar que

[...] a Ciência da Informação já se deu conta de que a disponibilidade de informação não será possível no futuro, se estiver sustentada pelos princípios pragmáticos tradicionais. (ZANDONADE, 2004, p. 14).

Na filosofia, Fuller (1999), por exemplo, acredita que trata-se de um movimento intelectual que ultrapassa barreiras disciplinares, tendo por objetivo reconstruir os problemas da epistemologia levando em conta que o conhecimento é social em sua essência. A epistemologia social reúne um conjunto de questões que teve sua primeira formulação relacionada à produção do conhecimento científico, porém incorporou, com o tempo, os conhecimentos em variadas dimensões públicas e multiculturais, assim como a conversão do conhecimento em tecnologia de informação, propriedade intelectual e apropriação social. Outro representante, Goldman (1999), reflete sobre qual seria a questão central para 
uma epistemologia social; se o conhecimento é uma propriedade do agente epistêmico, isolado do seu meio social, ou se ele envolve as relações entre esses agentes e suas circunstâncias sociais. Para buscar respostas para essa questão é preciso considerar o tipo de agente epistêmico, o tipo de conhecimento e o tipo de relações sociais que se observam. Ou seja, levar em conta que a situação epistêmica humana é, em grande parte, moldada por relações e instituições sociais. É por essa razão que a epistemologia social procura investigar os efeitos epistêmicos das interações sociais e dos sistemas sociais (GOLDMAN, 1999; GOLDMAN; BLANCHARD, 2015).

Assim, a epistemologia social teria sua razão de recuperação e presença dentro do campo de estudos da informação nos tempos atuais na finalidade de guiar as análises sobre as marcas sociais e epistêmicas fixadas nos dispositivos de informação e comunicação dos ambientes digitais.

\section{Dispositivos de informação e comunicação}

Estudos sobre as novas mídias digitais, na literatura das Ciências da Informação e da Comunicação (SIC) na França, procuram fundamentar uma economia política dos textos e das escritas na web. No recorte aqui apresentado, o interesse é o de refletir sobre a textualidade das práticas sociais, cognitivas e simbólicas, e dos gestos editoriais que conformam os dispositivos de informação e comunicação no meio digital.

Nesse sentido, vale recuperar o conceito de dispositivo segundo Foucault (1977), após ter sido reelaborado, nas pesquisas francesas das SIC, como um agenciamento de elementos que apresenta, entre outras, a particularidade de ter por objetivo articular meios em função de uma finalidade, ela própria ligada a uma situação que exerce constrangimentos e impõe limites (COUZINET, 2011; MARTELETO; COUZINET, 2012). Um dispositivo é, portanto, algo inscrito em um projeto, com uma missão ou finalidade a cumprir em uma situação particular, o que representa, tanto sua força fundante e razão de ser, quanto as limitações que pesam sobre os seus objetivos. Por outro lado, não é algo isolado, estando interligado a outros objetos da mesma natureza que lhe precedem ou su- 
cedem, fazendo assim parte de um conjunto de objetos e sentidos, todos atuando também enquanto dispositivos (METZGER, 2002). Para estudá-los é necessário desatar os elos que os exercem força sobre o conjunto assim constituído (COUZINET, 2009) levando em conta as suas dimensões, técnica e social, constituídas por elementos, indivíduos e conjuntos técnicos, tanto mais complexos quanto mais dependem da intervenção humana (SIMONDON, 1989).

Para a compreensão dos processos e práticas de informação e comunicação é oportuno lembrar que os dispositivos, tanto se expressam em sua dimensão coercitiva e de poder, inerente à vida social dos dispositivos em geral, quanto podem ser considerados como "seres culturais", isto é:

[...] um complexo que associa objetos materiais, textos, representações e que resulta na elaboração e no compartilhamento de ideias, de informações, de saberes, de julgamentos. Trata-se de configurações dinâmicas que traduzem a elaboração histórica dos recursos e das questões da cultura para uma sociedade: posturas, saberes, valores que não podem ser compreendidos de forma separada uns dos outros e que repousam sobre uma panóplia de objetos e de procedimentos, sem no entanto se reduzir a um único registro técnico. (JEANNERET, 2008, p. 16).

Uma das expressões sociais de um 'ser cultural' que tem um papel determinante na nossa sociedade seria, por exemplo, a imagem simbólica da ciência, ou da cientificidade. Sua simbologia se concentra sobre concepções, ainda que um tanto vagas, insistentes sobre o que são a precisão, a medida e a racionalidade, ideias que os números ou os algarismos, por exemplo, estão aptos a encarnar (JEANNERET, 2008).

Ao empregar a noção de "seres culturais" para a invenção e a reinterpretação dos dispositivos, trata-se de compreendê-los enquanto "compósitos" (LE MAREC, 2002), ou seja, como mistos de objetos, de representações e de práticas, que fornecem as suas referências de interpretação e de valor às formas culturais de uma sociedade, servindo como operadores de representações do mundo, de posturas culturais e de categorias de entendimento e de julgamento que uma cultura poderia reconhecer. 


\section{$4 \mathrm{O}$ meio ambiente e o modo de existência dos dispositivos enquanto 'seres culturais': a cultura digital}

A comunicação no meio ambiente virtual estaria associada à interatividade e à expressão da linguagem, enquanto a informação às formas escritas que asseguram a permanência dos registros, da memória e dos sinais de uso das mídias digitais mediadas pelo dispositivo técnico. Este possui mecanismos próprios para operar suas diferentes camadas de escrita: do sítio; do navegador; do software; do sistema de exploração do computador; dos servidores; dos protocolos de transmissão; dentre outros.

Sem essa interoperabilidade o dispositivo técnico não poderia funcionar, e, mesmo que funcionasse, o usuário não poderia entender os enunciados e as informações. Quem faz uso dessas mídias deve mobilizar, simultaneamente, o sentido de sua ação e um manejo mínimo do dispositivo, bem como dominar a técnica da escrita para inscrever o seu pensamento numa superfície de papel (DAVALLON, 2012a, 2012b). As 'oralidades escritas', ou 'escritas orais', pelas quais se organizam os processos de informação e comunicação nos espaços digitais, levam a se pensar em novas competências para agir e se expressar nos ambientes virtuais.

Por outro lado é importante refletir que os novos modos de comunicação não substituem nem marginalizam os precedentes, tendo em vista a recenticidade, em um panorama histórico, da implantação e universalização da escrita e da leitura nos moldes de comunicação das escolas, dos livros e das bibliotecas:

Do ponto de vista histórico, somente recentemente as sociedades, mesmo as mais avançadas, colocaram em prática uma política global de ensino da escrita e da leitura. (GOODY, 2007, p. 7).

Por isso, e apesar das mudanças aceleradas nos modos de informação e comunicação, o modelo escolar de conhecimento continua sendo o vetor principal da cultura informacional (MARTELETO, 2011). Os dispositivos de informação e comunicação são constituídos pelas relações entre os textos, os elementos técnicos que exercem a mediação e condicionam as escritas, e também pelos usos, que configuram a sua forma social (DAVALLON, 2012a; TARDY; JEANNERET, 2007). Eles funcionam nas mediações com outras mídias digitais, 
como os blogs, os sítios, as plataformas e redes de conversação, tendo como correlato a emergência de um novo "saber-ler-escrever", ou uma "competência digital", que não corresponde somente a conjuntos de normas que permitem manejar uma tecnologia, mas que constituem novos espaços de práticas e formas específicas de saberes no meio ambiente de uma cultura digital (DOUEIHI, 2008, p. 13-14; SANTOS, 2011). Que tipos de interações e colaborações os dispositivos digitais estimulam, acrescentam, substituem ou obstruem? Como se inserem e complementam outros espaços de informação e comunicação online e offline? (GARTON; HAYTHORNWAITE; WELLMAN, 1997; RECUERO, 2012).

Para refletir sobre essas questões é preciso elaborar uma compreensão do que seria uma "cultura digital" inscrita numa dimensão temporal e histórica, com a finalidade, não de reificá-la, e sim de obter elementos sobre a plasticidade e as interpretações possíveis de suas composições. Sobre esse propósito, se questiona se a cultura digital seria uma nova cultura ou se corresponderia ao aporte das tecnologias e de seu "impacto" na nova configuração do mundo cultural. Diante dessas questões que se complementam, caberia interrogar ainda:

[...] a partir de qual momento e sob quais condições, esses usos das mídias informatizadas foi acompanhado pelo surgimento de modos de fazer e de objetos culturais até então desconhecidos? Nós nos alternamos entre o impacto do digital sobre a cultura e a questão da constituição de uma 'cultura digital. (DAVALLON, 2012b, doc. não paginado).

Monnoyer-Smith (2013, p. 12-13), ao considerar a web como um espaço de expressão e movimento de uma cultura digital, lembra que ela se caracteriza por sua opacidade sociotécnica, a qual contribui para estruturar as mediações, tanto em seu interior (mediações online), quanto em seu exterior (mediações offline). O próprio espaço web-digital resulta desse conjunto de mediações, que permite traçar os seus contornos técnicos particulares, funcionais e de uso. A mesma autora lembra que existem duas tendências correntes que orientam a compreensão desse espaço: a reificação e a naturalização. A primeira, a reificação, consiste em conceber a web como uma realidade monolítica, externa aos atores, que utilizam-na como um objeto neutro e atribuem-lhe um sentido através da sua instrumentalização sociosemiótica. A segunda tendência trata da na- 
turalização, que seria entender a web como o nivelamento das relações de mediações a partir das quais ela se formou, desconsiderando o conjunto heterogêneo e evolutivo de seus componentes, sejam eles técnicos ou sociais. Uma via terceira para estudar o espaço da web como local de exercício de uma "cultura digital" partiria do entendimento de que aquilo que está "dobrado" na web e permanece invisível na superfície dos escritos na tela, é um construto sociotécnico que convém desdobrar para tornar suas camadas e composições inteligíveis (MONNOYER-SMITH, 2013, p. 12-14).

Por outro lado, estudos sobre comunidades virtuais relatam que, em geral, as relações offline afetam as relações online. Sobre as mídias de conversação, as pesquisas indicam que certo grau de enraizamento no grupo social estimula o comportamento de postagem do participante, resultando em um número maior de postagens. No entanto, as pesquisas não analisam se e como isso afeta o conteúdo da discussão, ou as dificuldades de gerenciamento e moderação das mensagens e dos debates (GARTON; HAYTHORNTHWAITE; WELLMAN, 2007; HAYTHORNTHWAITE, 2008). Quanto às listas de discussão ou comunidades virtuais, por exemplo, algumas encontram-se incorporadas em comunidades bem integradas, estimulando ou desdobrando-se em redes informais densas entre os coletivos e grupos, e podendo, assim, colaborar com a expansão das redes dos participantes, apesar de todos os fatores contextuais que afetam a colaboração. Portanto, deve-se distinguir entre os diferentes tipos de listas de discussão virtuais: as híbridas e enraizadas em comunidades; e as puras, somente virtuais (MATZAT, 2009). Do ponto de vista interativo e de funcionalidade técnico-semântico-social, os estudos apontam certos aspectos negativos, como a falta de confiabilidade nas pessoas e em suas mensagens; o emprego do tempo requerido para participação; ou o agravamento de overload de informações por usuário. Os aspectos positivos estariam associados principalmente às possibilidades de se obter informações e manter-se atualizado, ainda que considerando o problema frequente das mensagens off-topic - questões postadas que não se relacionam com os propósitos da lista representante de uma comunidade (MATZAT, 2009). 
Para quem se dispõe a entender o funcionamento de um coletivo e sua atividade nos espaços digitais, trata-se de perceber o que eles são na vida cotidiana e o modo com que podem se transformar na mediação das redes técnicas, observando-se tanto o caráter instrumental quanto o caráter substantivo das interações, compartilhamentos e mobilizações. Dessa forma, um dispositivo na web não existe nele mesmo, mas nas mediações dos processos de informação e comunicação no espaço virtual e das mudanças na ordem social. No caso dos coletivos mobilizados em redes de participação social, interessa analisar as formas combinatórias, presenciais e virtuais, de participação e diálogo, com base nas seguintes questões: de que forma a comunidade utiliza, em suas práticas, aquilo que é elaborado coletivamente? As trocas entre os membros da lista de discussão podem constituir um corpus de novos saberes para os atores? Para tanto o olhar infocomunicacional dirigido para o objeto "comunidade online" deveria observar e analisar a dinâmica das relações, os jogos e estratégias dos atores, as práticas sociais, as trocas e as representações.

\section{O social e o textual na cultura digital - o 'arquitexto'}

Davallon et al. (2003) ressaltam que a noção de texto não pode ser definida apenas como semiótica (sistema de signos), uma vez que, para se tornar um "texto", o objeto deve ser reconhecido socialmente como tal. Sua construção está intimamente associada à materialidade do objeto e ao modo como ele é construído e usado em uma determinada sociedade. O texto possuiria, assim, as dimensões semiótica e simbólica, e a tensão constante entre a homogeneidade e heterogeneidade apontaria para uma importante característica intrínseca deste objeto: a sua dupla face, ou seu paradoxo. É possível, assim, defini-lo como uma estrutura de enunciados - orais, escritos, imagéticos, sonoros - que podem ter sua virtualidade expandida.

Por outro lado, o termo hipertexto surge antes da criação da web, em 1965, referindo-se a uma forma não linear de apresentar a informação textual. Trata-se de uma rede de remissões internas ao texto em reconstrução permanente que, possuindo unidades básicas, permite a construção de elos conceituais 
entre textos e iconografias paralelas, a associação a outros dados e a integração entre palavras e imagens.

A corporificação do hipertexto se dá somente com o advento da web. Nesta perspectiva, o hipertexto pode ser entendido como um sistema reticular, distribuído, aparentemente infinito, que depende da habilidade do "leitor" de manipular seus elos conceituais e estabelecer ligações lógicas de significação entre diferentes textos ocultos, e que exprime os interesses e os conhecimentos de uma comunidade em tempo real.

Esta materialização do hipertexto na web levou um grupo de pesquisadores, dentre eles Emmanuel Souchier, Yves Jeanneret, Jean Davallon, Joëlle Le Marec, e Cécile Tardy, a realizar estudos teóricos e aplicados a respeito de uma nova arquitetura textual, nomeada de 'arquitexto'. Segundo eles, aquilo que se vê representado na tela, no texto ou na imagem é regido por uma combinação de algoritmos, um código matemático que geralmente permanece escondido ou invisível em outra dimensão desta estrutura. O arquitexto, segundo Davallon (2012a), é uma ferramenta de engenharia textual híbrida, herdeira da computação, da lógica e da linguística, que permite fazer o elo entre as linguagens técnica e simbólica e que rege os mais diversos circuitos do texto digital (redação, edição, leitura, etc.). Assim, a noção de arquitexto designa os objetos informáticos capazes de reger a escrita e fornecer seus formatos e seus recursos: por exemplo, o tratamento do texto fornece os recursos para a redação, o software de mensagens oferece o enquadramento para as trocas, os motores de busca possibilitam o acesso aos textos: "Um arquitexto é uma escrita da escrita, uma escrita que condiciona outras escritas.” (TARDY; JEANNERET, 2007, p. 24).

Para compreender o modo de operação dos arquitextos em contextos específicos, é necessário estudar o seu papel de “[...] operadores do encontro entre mediações técnicas (quais ferramentas?), mediações sociais (quais coletivos?) e mediações escritas (quais textos?)." (TARDY; JEANNERET, 2007, p. 32). Em qualquer caso é relevante considerar que não é o limite de uma estrutura organizacional ou um campo que prevalece nos estudos sobre os arquitextos, mas a circulação dos objetos sócio-semióticos em espaços diferenciados. Para uma análise aprofundada desses artefatos, busca-se evidenciar como estes compor- 
tam: “[...] uma escrita das práticas, os recursos semióticos dessa representação, a maneira como o arquitexto, pela sua dimensão operacional, proporciona, por outro lado, a antecipação de usos possíveis [...]” (TARDY, JEANNERET, 2007, p. 32).

Bonaccorsi (2013) reflete que o termo 'interface', utilizado na linguagem informática e no senso comum, não é suficiente para apreender a dimensão semiótica da estrutura textual das ferramentas de escrita na web, as quais integram um imaginário da comunicação, mas igualmente prevêem e configuram a escrita dos 'escritos de tela' (écrits d'écran). Segundo a autora, "a noção de arquitexto (do grego arché, origem e ordem) é útil para qualificar a dupla função dessas ferramentas: produzir o texto e torná-lo visível.” (BONACCORSI, 2013, p. 132). O arquitexto é, pois, uma forma de representar o 'universo do software' necessário para a produção de um texto, ressaltando que os softwares permitem tanto a escrita quanto a apresentação e a visualização das formas textuais que se reproduzem e se transformam:

Desse modo, a dimensão modeladora do software não diz respeito somente à formatação do texto: o uso modifica materialmente este texto. A noção de arquitexto tem como função descrever a dualidade específica dos textos informatizados, entre modelo e invenção. (BONACCORSI, 2013, p. 13).

As ideias de 'modelo' e 'invenção', associadas ao conceito de hipertexto retomam, pois, a arché fundamental e sempre presente nos textos, referindo-se tanto a uma arquitetura informacional técnica e simbólica, quanto à memória e aos traços de autoria, mediações, apropriações e usos sociais dos textos, constituindo assim um arquivo textual em constante reconfiguração. Seja qual for a terminologia descritiva empregada para um modelo epistemológico orientador dos estudos das formas textuais na cultura digital:

[...] o fato é que o texto informacional - menos estruturado ou sintaticamente hierarquizado do que o texto tradicional, em que a subjetividade do leitor era estável e centrada em coordenadas culturais que buscavam garantir a univocidade da palavra impressa - convida hoje o sujeito a tatear em novos 'sensores' necessários à sua orientação. (SODRÉ, 2012, p. 11). 
Para entender essas novas textualidades, os estudos da informação e comunicação precisam se orientar por uma matriz cognitiva e um modelo epistemológico que aproximem o sentido de permanência e organização dos textos às mediações, interações e apropriações necessárias à sua produção, acesso e expressão.

\section{Notas finais}

A retomada dos construtos de dois dos fundadores do campo de estudos e práticas da informação - Jesse Shera e Margareth Egan - indica que os princípios da epistemologia social e sua atualização nos dias de hoje serviriam como um quadro de fundo para a leitura informacional dos processos de produção, mediação e apropriação social dos conhecimentos. E, além disso, serviriam ainda para a reunião interdisciplinar de uma pauta de questões que as Ciências Humanas e Sociais, dentre elas, a Informação e a Comunicação, precisam enfrentar no estudo das novas práticas de escrita e leitura na cultura digital.

No caminho reflexivo a respeito dos dispositivos de informação e comunicação numa cultura digital, considerou-se a web não somente como uma mídia na qual os textos recebem uma forma editorial, mas também como um lugar onde certos imaginários participam, pela escrita, de um espaço narrativo que, apesar de estar tecnicamente interconectado, permanece profundamente heterogêneo (DAVALLON, 2012b). Diante dessa dispersão e variedade, de que forma pode-se estudar esses arranjos textuais de modo tal que suas marcas sociais e seus contextos de produção se façam notar? Quais seriam as mediações atuantes na representação arquitextual, relacional e interativa?

O foco é dessa forma orientado para a maneira como se atualiza uma forma de escrita e de manipulação de textos a partir de saberes compartilhados, tornando visível uma teia de práticas e sentidos evocada no instante das leituras e apropriações. Por isso, a análise não pode ser conduzida em termos da recepção de uma obra ou produto já realizado, e sim de textos, gestos editoriais e valores que conformam os 'seres culturais'. É necessário então questionar a tradicional classificação dos textos e gêneros, sem, no entanto, perder de vista sua 
permanência e seus significados no imaginário social e cultural. Nesse mesmo sentido, questiona-se também certa 'personificação da recepção' para interessarse pelos objetos textuais produzidos pelos atores, pelas mediações, assim como pela circulação dos textos (SOUCHIER et al., 2007).

O lugar determinante dado à materialidade dos dispositivos, de suas formas textuais e de suas marcas relacionais, sociais, cognitivas e semânticas não caracteriza, como uma abordagem tecnicista, o tratamento dado ao tema dos dispositivos de informação e comunicação. Diferentemente, continua sendo uma abordagem sócioantropológica que se defronta com as intensas transformações das mídias na cultura digital.

\section{Referências}

BONACCORSI, J. Approches sémiologiques du web. In: BARATS, C. Manuel d'analyse du web. Paris: Armand Colin, 2013. p. 125-146.

COUZINET, V. Les dispositifs: question documentaire. In: GARDIÈS, C. (Org.). Approche de l'information-documentation: concepts fondateurs. Toulouse: Cépaduès-Éditions, 2011.

COUZINET V. Dispositifs info-communicationnels: contributions à une définition. In: COUZINET, V. (Org.). Dispositifs info-communicationnels: questions de médiations documentaires. Paris: Hermès-Lavoisier, 2009. p. 19-30.

DAVALLON, J. Traces d'usage dans un corpus de sites de tourisme. In: DAVALLON, J. L'économie des écritures sur le web. Paris: Hermès ScienceLavoisier, 2012a. v. 1.

DAVALLON, J. Du numérique pour la culture à la culture numérique? In: JORNADA CIENTÍFICA INTERNACIONAL DA REDE MUSSI, 2., 2012, Rio de Janeiro. Anais... Rio de Janeiro: Rede Mussi, 2012b.

DOUEIHI, M. La grande conversion numérique. Paris: Ed. du Seuil, 2008.

EGAN, M. E.; SHERA, J. H. Foundations of a theory of bibliography. Library Quarterly, Chicago, v. 22, p. 125-137, 1952. 
FOUCAULT, M. Le jeu de Michel Foucault [entretien avec D. Colas et al.]. Ornicar? Bulletin Périodique du Champ Freudien, [S.1.], n. 10, p. 62-93, jul., 1977.

FULLER, S. Social epistemology. In: BULLOCK, A.; TROMBLEY, S. (Ed.). Norton Dictionary of Modern Thought. New York: Norton, 1999. p. 801-802

GARTON, L.; HAYTHORNTHWAITE, C.; WELLMAN, B. Studying on-line social networks. Journal of Computer Mediated Communication, Hoboken, n. 3, 2007.

GOLDMAN, A. Knowledge in a Social World. Oxford: Oxford University Press, 1999.

GOLDMAN, A.; BLANCHARD, T. Social Epistemology. In: ZALTA, N. (Ed.) The Stanford Encyclopedia of Philosophy. Stanford: Centre for the Study of Language and Information, 2015.

GOODY, J. L'oralité et l'écriture. Communication et Langages, [S.1.], n. 154, 2007.

HAYTHORNTHWAITE, C. Learning relations and networks in web-based communities. International Journal of Web Based Communities, Olney, v. 4, n. 2, p. 140-158, 2008.

JEANNERET, Y. La relation entre médiation et usage dans les recherches en information-communication. In: COLÓQUIO INTERNACIONAL MEDIAÇÕES E USOS DE SABERES E INFORMAÇÕES: UM DIÁLOGO FRANÇABRASIL, 1., 2008, Rio de Janeiro. Anais... Rio de Janeiro: Rede Mussi, 2008. p. $37-59$

LE MAREC, J. Ce que le terrain fait aux concepts: vers une théorie des composites. Habilitation à diriger des recherches (HDR). Paris: Université Paris 7, Cinéma, communication et information, 2002.

MARTELETO, R. M. À quoi servent les recherches? In: GARDIÈS, C. (Org.). Approche de l'information-documentation: concepts fondateurs. Toulouse: Ed. Cépaduès, 2011. p. 229-232.

MARTELETO, R. M.; COUZINET, V. Mediações e dispositivos de informação e comunicação na apropriação de conhecimentos: elementos conceituais e empíricos a partir de olhares intercruzados. RECIIS: Revista Eletrônica de Comunicação, Informação \& Inovação em Saúde, Rio de Janeiro, v. 7, p. 1-16, 2012. 
MATZAT, U. Quality of Information in Academic E-mailing lists. Journal of the American Society for Information Science and Technology, Hoboken, v. 60, n. 9, p. 1859-1870, 2009.

METZGER, J. P. Les trois pôles de la Science de l'information. Actes du Colloque International MICS-LERASS Recherches Récentes en Sciences de 1 'Information: convergences et dynamiques. Toulouse, 21-22 mars 2002, ABBS, p. 17-28, 2002.

MONNOYER-SMITH, L. Le web comme dispositif: comment appréhender le complexe? In: BARATS, C. Manuel d'analyse du web. Paris: Armand Colin, 2013, p. 12-30.

RECUERO, R. A conversação em rede: comunicação mediada pelo computador e redes sociais na Internet. Porto Alegre: Sulina, 2012.

SANTOS, L. G. Politizar as novas tecnologias: o impacto sociotécnico da informação digital e genética. São Paulo: Editora 34, 2003.

SHERA, J. H. "Philosophy of Librarianship". In: WEDGEWORTH, Roberto (Ed). ALA World encyclopedia of library and Information services. Chicago: ALA, 1980, p. 314- 317.

SHERA, J. H. Epistemologia Social, Semântica Geral e Biblioteconomia. Ciência da Informação, Rio de Janeiro, v. 6, n. 1, p. 9-12, 1977.

SHERA, J. H. The sociological relationships of information science. Journal of the American Society for Information Science, Hoboken, v. 22, n. 1, p. 76-80, mar./apr., 1971.

SHERA, J. H. Toward a theory of Librarianship and Information Science. Ciência da Informação, Brasília, v. 2, n. 2, p. 87-96, 1973.

SIMONDON, J. J. Du mode d'existence des objets techniques. Paris: Aubier, 1989.

SOUCHIER, E. et al. Formes et pouvoirs de l'énonciation éditoriale. Communication et Langages, [S.1.], v. 154, p. 23-38, 2007.

SODRÉ, M. Prefácio. In: REGIS, F. et al. (Org.). Tecnologias de comunicação e cognição. Porto Alegre: Sulina, 2012. p. 9-12.

TARDY, C.; JEANNERET, Y. (Coord.). L’écriture des médias informatisés. Paris: Hermès Science-Lavoisier, 2007.

ZANDONADE, Tarcísio. Social epistemology from Jesse Shera to Steve Fuller. Library Trends, Baltimore, v. 52, n. 4, p. 810-832, spring 2004. 


\title{
The social epistemology and the digital culture: thoughts sur- rounding the types of writings on the web
}

\begin{abstract}
The article presents some theoretical and methodological lines to reflect on the production, mediation and appropriation of knowledge and the configuration of information and communication web devices, within the context of digital literacy. Therefore, it recovers the idea of social epistemology, coined by two founders of information studies field, Shera J. and M. Egan, updated by authors of information and philosophy, in order to reflect on how the epistemic forms are related to social and institutional practices. This was considered a promising avenue to explore and understand the ways of being of digital spaces and their media. Finally, we explore some studies of the French line of Information and Communication Sciences, specifically the concepts of infocommunicational device; digital culture; text; hypertext; and architext, in order to reflect on the structural forms of writings and lectures on the web. If the social epistemology directs a study program on the issues of access, production and dissemination of knowledge, the research on forms of writing indicates theoretical and practical ways to understand and practice the writings on the web.
\end{abstract}

Keywords: Social epistemology. Information and communication devices. Digital culture. Architext.

Recebido em 21/09/2015

Aceito em 20/12/2015 